\title{
A Reduced Order Model of Two-Phase Flow, Heat Transfer and Combustion in Circulating Fluidized-Beds
}

\author{
Annual Report \\ Reporting Period Start Date: 10/01/01 \\ Reporting Period End Date: 9/30/02 \\ Principal Investigator: Paul Cizmas \\ Date Report was Issued: December 2002 \\ DOE AwARD Number: DE-FC26-00NT40903 \\ Texas Engineering Experiment Station \\ OfFice of Sponsored Research \\ 332 Wisenbaker Engineering Research Center, 3000 TAMU \\ College Station, TX 77843-3000
}

\author{
AND \\ Antonio Palacios \\ San Diego State University \\ San Diego, CA 92182-7720
}




\section{Disclaimer}

This report was prepared as an account of work sponsored by an agency of the United States Government. Neither the United States Government nor any agency thereof, nor any of their employees, makes any warranty, express or implied, or assumes any legal liability or responsibility for the accuracy, completeness, or usefulness of any information, apparatus, product, or process disclosed, or represents that its use would not infringe privately owned rights. Reference herein to any specific commercial product, process, or service by trade name, trademark, manufacturer, or otherwise does not necessarily constitute or imply its endorsement, recommendation, or favoring by the United States Government or any agency thereof. The views and opinions of authors expressed herein do not necessarily state or reflect those of the United States Government or any agency thereof. 


\section{Abstract}

This report summarizes the objectives, tasks and accomplishments of the second year of this research project. The report presents the following program deliverables: (1) visualization tools for reconstructing simulated data; (2) algorithms for reducing the partial differential equations to ordinary differential equations; and (3) visualization tools for Galerkin ordinary differential equations. 


\section{Contents}

1 Introduction $\quad 5$

$\begin{array}{llr}2 & \text { Executive Summary } & 6\end{array}$

$\begin{array}{llr}3 & \text { Experimental } & 7\end{array}$

4 Results and Discussion $\quad 7$

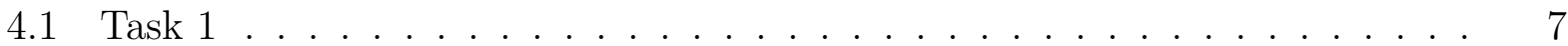

4.2 Task $2 \ldots \ldots \ldots \ldots \ldots$

4.3 Task $3 \ldots \ldots \ldots \ldots \ldots$

5 Conclusions $\quad 13$

\section{List of Figures}

1 Time coefficients and reconstructed solutions with ODEx . . . . . . . . 14

2 Geometry and boundary conditions for case s3b. . . . . . . . . . . . . 15

3 Cumulative sum of energy of POD modes for case s3b. . . . . . . . . . . . 15

4 Velocity variation at point A, case s3b. . . . . . . . . . . . . . 16

5 Velocity variation at point B, case s3b. . . . . . . . . . . . . . 16

6 Reconstructed $u(0.5, t)$ at different tolerance levels. . . . . . . . . . . . . . 17

7 Velocity variation at point A, case s3c. . . . . . . . . . . . . 18

8 Velocity variation at point B, case s3c. . . . . . . . . . . . 18

\section{List of Tables}

1 Values of left-hand-side and right-hand-side terms of the $x$-momentum conservation equation at different grid locations in MFIX and in the low order model. . . . . . . . . . . . . . . . . . . . . 10

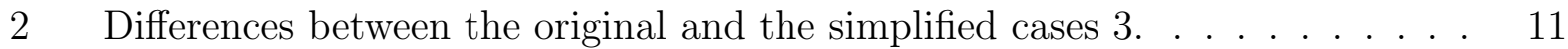

3 Tolerance and error levels in Burgers equation. . . . . . . . . . . . . . . . 12 


\section{Introduction}

This report presents the research activity completed during the second year of the project. The report describes the tasks assigned for the reviewing period, the accomplishments and challenges of this period, as well as the results obtained so far. The section Results and Discussion briefly presents the research objectives of the second year of the project and describes how these tasks were accomplished. This section also present a sample of the results obtained. There were no changes in the approach originally proposed to complete the research program, although additional methodologies have been explored in order to implement the algorithm for reducing the partial differential equations (PDEs) to ordinary differential equations (ODEs). 


\section{Executive Summary}

The modeling of dense multiphase flow reactors is crucial for circulating fluidized-bed combustors and fluid catalytic cracking risers. The governing equations describing the phenomena inside multiphase flow reactors consist of a system of highly coupled partial differential equations. Solving these equations is a difficult and computing intensive task. One of the goals of this project is to reduce the computational effort by developing a low order model based on the proper orthogonal decomposition (POD) method. Another goal of this project is to develop a visualization package for the analysis of transport phenomena, as well as for the storage of the information produced by numerical simulation and/or experimental investigation.

This report presents the research activity completed during the second year of the project. The report describes the tasks assigned for the reviewing period, the accomplishments and challenges of this period, as well as the results obtained as of September 2001. The goals of the project during the second year were to develop (1) visualization tools for reconstructing simulated data; (2) algorithms for reducing the partial differential equations to ordinary differential equations; and (3) visualization tools for Galerkin ordinary differential equations.

The first task, the development of visualization tools for reconstructing simulated data, has been completed. This included visualization of reconstructed flow motion, and visualization of flow motion in phase space through POD time coefficients. Using POD spatial modes, visualizations of the reconstructed flow motion were generated. POD amplitude coefficients were used to create animations of the temporal behavior of the flow in phase space.

The second task, the development of an algorithm for reducing the partial differential equations (PDEs) to ordinary differential equations (ODEs), has been completed. During this task several implementations of the low order models have been developed. The main implementations included: (1) ODEx, a low order model that followed MFIX discretization, although required reorganization of the discretized equations before projection on the basis functions; (2) ODExNL, a low order model implementation similar to ODEx, in which the system of ODEs has been transformed into a non-linear system of equations; (3) ODExMIN, a low order model implementation in which instead of projecting the PDEs on the basis functions, the time coefficients were solved using an error minimization methodology; and (4) ODExMFIX, a low order model in which the implementation closely followed the MFIX code.

The third task, the development of visualization tools for Galërkin ODEs has been started. This included visualization of reconstructed flow motion, and visualization of flow motion in phase space through POD time coefficients. Using POD spatial modes, visualizations of the reconstructed flow motion were generated. POD amplitude coefficients were used to create animations of the temporal behavior of the flow in phase space. This task is scheduled to end on December 2002. 


\section{Experimental}

No experimental work has been planned or completed during this reporting period.

\section{Results and Discussion}

The following three tasks have been planned for the second year period:

\begin{tabular}{|l|l|l|l|l|}
\hline \hline & Program deliverables & Begin date & End date & Status \\
\hline 1. & Develop visualization tools for reconstructing simulated data & $04 / 01 / 01$ & $12 / 31 / 01$ & Completed \\
\hline 2. & Develop and implement algorithm for reducing the PDEs to ODEs & $10 / 01 / 01$ & $09 / 30 / 02$ & Completed \\
\hline 3. & Develop visualization tools for Galërkin ODEs & $04 / 01 / 02$ & $12 / 31 / 02$ & In progress \\
\hline \hline
\end{tabular}

\subsection{Task 1}

The work on the visualization tools for reconstructing simulated data has been completed. This included visualization of reconstructed flow motion, and visualization of flow motion in phase space through POD time coefficients. Using POD spatial modes, visualizations of the reconstructed flow motion were generated. POD amplitude coefficients were used to create animations of the temporal behavior of the flow in phase space.

The following computer codes written in Matlab have been developed for this task:

animate.m Creates an MPEG movie of a spatio-temporal variable of the form $u(x, t)$, where $x$ represents space and $t$ time. Usage: Within MATLAB, first execute "animate" then "mpegwrite(M,colormap,'file.mpeg')" Input: *.dat file containing individual values of $u(x, t)$ at times $t 1, \ldots, t_{N}$. Output: "file.mpeg" containing movie in MPEG format.

vis_3dphase.m Creates various 3D phase-space projections of the POD time-series coefficients obtained from the POD decomposition of $u(x, t)$. Usage: Within MATLAB, execute "vis_3dphase". Input: "fileproj*.ascii" files containing POD time coefficients. Output: MATLAB graph with various 3D phase-space projections. This graphic file can then be saved in various other formats such as postscript, gif, etc.

vis_modes2.m Displays a static plot of the most relevant POD modes found from the POD decomposition of $u(x, t)$. Usage: Within MATLAB, execute "vis_modes2". Input: "filemode*.dat" files containing POD eigenfunctions. Output: MATLAB graph depicting the time-average of $u(x, t)$ and the most energetic POD modes. This graphic file can then be saved in various other formats such as postscript, gif, etc.

vis_pod_rec.m Generates an MPEG movie of the dynamics of $u(x, t)$ reconstructed via its POD decomposition. Usage: Within MATLAB, first execute "vis_pod_rec" then "mpegwrite(M,colormap,'file.mpeg')" Input: "fileproj*.ascii" files containing POD time 
coefficients. "filemode*.dat" files containing POD eigenfunctions. Output: "file.mpeg" containing movie in MPEG format.

vis_snapshots.m Displays 10 snapshots of a spatio-temporal variable $u(x, t)$. Usage: Within MATLAB, execute "vis_snapshots". Input: "*.dat file containing individual values of $u(x, t)$ at times $t 1, \ldots, t_{N}$. Output: MATLAB graph with 10 time-snapshots. This graphic file can then be saved in various other formats such as postscript, gif, etc.

vis_spectrum.m Creates a bar plot of POD energy spectrum contained within each mode as well as cumulative POD energy. Usage: Within MATLAB, execute "vis_spectrum. Input: "filespectrum.dat" file containing POD energy spectrum of $u(x, t)$. Output: MATLAB graph with POD energy spectrum. This graphic file can then be saved in various other formats such as postscript, gif, etc.

vis_time_series.m Generates in a single plot a graph of the POD time-series coefficients associated with the ten most energetic POD eigenfunctions. Input: "fileprojection*.ascii" files containing POD time coefficients. Output: MATLAB graph with POD time series. This graphic file can then be saved in various other formats such as postscript, gif, etc.

ani_set1.m Creates MPEG movie showing original animation, POD reconstruction using 3 modes, and animation of 3D phases-space A red dot moves through phase-space showing time evolution. Usage: Within MATLAB, execute "ani_set1" then "mpegwrite(M,colormap,'file.mpeg')". Input: "fileproj*.ascii" files containing POD time coefficients. "filemode*.dat" files containing POD eigenfunctions. Output: "file.mpeg" containing movie in MPEG format.

ani_set2.m Creates MPEG movie showing several POD reconstructions with different number of modes, and simultaneously, a red dot moves along the time series coefficients to illustrate the relationship between time-series values and POD reconstructions. Usage: Within MATLAB, execute "ani_set2" then "mpegwrite(M,colormap,'file.mpeg')". Input: "fileproj*.ascii" files containing POD time coefficients. "filemode*.dat" files containing POD eigenfunctions. Output: "file.mpeg" containing movie in MPEG format.

ani_set3.m Generates MPEG moving illustrating time evolution in phase-space together with POD reconstruction. Usage: Within MATLAB, execute "ani_set3" then "mpegwrite(M,colormap,'file.mpeg')". Input: "fileproj*.ascii" files containing POD time coefficients. "filemode*.dat" files containing POD eigenfunctions. Output: "file.mpeg" containing movie in MPEG format.

ani_set4.m Generates MPEG movie of MFIX simulation, and tine-series values animated as bar plots, together with POD reconstruction. Usage: Within MATLAB, execute "ani_set4" then "mpegwrite(M,colormap,'file.mpeg')". Input: "fileproj*.ascii" files 
containing POD time coefficients. "filemode*.dat" files containing POD eigenfunctions. Output: "file.mpeg" containing movie in MPEG format.

ani_set5.m Creates simultaneous animations with original MFIX simulations, and successive POD reconstructions. Usage: Within MATLAB, execute "ani_set5" then "mpegwrite(M,colormap,'file.mpeg')". Input: "fileproj*.ascii" files containing POD time coefficients. "filemode*.dat" files containing POD eigenfunctions. Output: "file.mpeg" containing movie in MPEG format.

Part of these results were presented at the Multiphase Fluid Dynamics Research Consortium meeting in Columbia, MD, September 2002 and at the "Vision 21" meeting held at Iowa State University in November 2002. This task was completed by Dr. Antonio Palacios of the Mathematics Department at San Diego State University.

\subsection{Task 2}

The development of an algorithm for reducing the partial differential equations (PDEs) to ordinary differential equations (ODEs) started by using the Navier-Stokes equations, a subset of the equations used in the MFIX code. In the beginning, two approaches have been used for the derivation of the projected equations of mass, momentum and energy conservation: (1) using the governing equations written in primitive variables, and (2) using the governing equations written in conservative variables. It was concluded that using the primitive variables was the best option because this approach avoids quotients of sums. The sums were the result of replacing the flow variables by their POD decomposition.

Once it was decided to use primitive variables, several approaches have been used to project the PDEs onto the basis functions. In a first trial, the PDEs of the governing equations (mass and momentum for gas only) were projected without taking into account the MFIX implementation, that is, using a discretization that did not take into account the upwinding direction. The time coefficients obtained by solving the ODE system were compared against the time coefficients obtained directly from MFIX using the properties of the basis functions. The agreement was good only for a short period of time at startup.

The difference between the MFIX solution and the reconstructed solution was attributed to the fact that (1) the projected ODEs did not take into account upwinding, and (2) the ODEs were obtained using a differential form of the governing equations while MFIX used an integral form of the governing equations. In addition, an investigation of the local accuracy of the MFIX results was done using the subroutine CHECK_CONVERGENCE provided by Dr. Madhava. It was found that the level of the local errors varied between 2 and $100 \%$. These levels of the local error do not preclude the MFIX code from converging globally, but they could be a factor in altering the reconstructed solution.

The accuracy problem was further investigated. In order to generate the system of ODEs, the governing equations in MFIX must be rearranged. For example, the conservation 
of momentum in the $x$-direction, which in MFIX is written as

$$
a_{p} u_{p}=\sum_{n b} a_{n b} u_{n b}+b-\left(P_{E}-P_{W}\right) \Delta y
$$

must be rearranged in the low order model as

$$
\rho_{p}^{\text {old }} \Delta V \frac{u_{p}-u_{p}^{\text {old }}}{\Delta t}=\sum_{n b} a_{n b}\left(u_{n b}-u_{p}\right)-\left(P_{E}-P_{W}\right) \Delta y .
$$

Values of the left-hand-side (LHS) and right-hand-side (RHS) terms of equations (1) and (2) are shown in Table 1.

\begin{tabular}{cccc}
\hline \multicolumn{2}{c}{ MFIX } & \multicolumn{2}{c}{ Low Order Model } \\
LHS & RHS & LHS & RHS \\
\hline-.839 & -.827 & $-.136 \mathrm{E}-01$ & $-.180 \mathrm{E}-02$ \\
-.778 & -.767 & $-.127 \mathrm{E}-01$ & $-.168 \mathrm{E}-02$ \\
-.715 & -.705 & $-.117 \mathrm{E}-01$ & $-.154 \mathrm{E}-02$ \\
-.650 & -.641 & $-.106 \mathrm{E}-01$ & $-.141 \mathrm{E}-02$ \\
-.583 & -.575 & $-.956 \mathrm{E}-02$ & $-.126 \mathrm{E}-02$ \\
-.514 & -.507 & $-.843 \mathrm{E}-02$ & $-.111 \mathrm{E}-02$ \\
-.766 & -.755 & $-.124 \mathrm{E}-01$ & $-.164 \mathrm{E}-02$ \\
-.711 & -.701 & $-.115 \mathrm{E}-01$ & $-.153 \mathrm{E}-02$ \\
\hline
\end{tabular}

Table 1: Values of left-hand-side and right-hand-side terms of the $x$-momentum conservation equation at different grid locations in MFIX and in the low order model.

The projection of the discretized equations consists basically of multiplying each LHS and RHS terms by the components of the POD basis vector and adding them. Although the difference between the LHS and RHS terms in the two columns is the same, the form used in the low order model for projections produces inaccurate results.

The code developed to project the PDEs onto the basis functions and to solve the ODEs that resulted was called ODEx. To verify that the ODEx produced correct results, two additional approaches have been developed and implemented. In the first approach, the system of ODEs has been transformed into a non-linear system of equations. The computer code that implemented this approach was called ODExNL. In the second approach, the PDEs have been transformed into a system of ODEs by replacing the dependent variables by the POD decomposition, e.g., $u(x, t)$ was replaced by $\sum_{i}^{m} a_{i}^{u} \phi_{i}^{u}$, where $m$ is the number of modes. Instead of projecting the ODEs onto the basis functions $\phi_{i}$, the ODEs have been solved by using a minimization process, similar to the approach used to solve a linear system of NE equations with NU unknowns, where NE $>$ NU. The computer code that implemented this approach was called ODExMIN. 


\begin{tabular}{lllll}
\hline & Original & & Simplified & \\
& Case 3 & Case s3a & Case s3b & Case s3c \\
\hline IMAX & 124 & 20 & 40 & 40 \\
JMAX & 108 & 20 & 50 & 40 \\
XLENGTH [cm] & 39.37 & 1.0 & 10.0 & 10.0 \\
YLENGTH [cm] & 58.44 & 1.0 & 10.0 & 10.0 \\
BC_V_g [cm/s] & $28.4 / 998.8 / 28.4$ & $2.84 / 10.8 / 2.84$ & $2.84 / 10.8 / 2.84$ & $1.0 / 20.8 / 1.0$ \\
DISCRETIZE & Superbee & Central Differencing & Central Differencing & Superbee \\
\hline
\end{tabular}

Table 2: Differences between the original and the simplified cases 3.

The two new approaches for solving the time coefficients were applied to the simplified case 3. Note that three simplified case 3 were used in the analysis. The differences between case 3 and the simplified versions are shown in Table 2.

The results of the two codes ODExNL and ODExMIN were in agreement to the results obtained using ODEx. The reconstructed solutions, however, were in good agreement with the MFIX results only for small values of time. A significant difference between the reconstructed results and the MFIX solution has been obtained as time increased. The time coefficients and reconstructed velocities with ODEx are shown in Fig. 1, where the Poddec data represent the time coefficients calculated using the MFIX results.

The first four plots in Fig. 1 show that the time coefficients obtained from ODEx captured approximately the shapes of the Poddec data. The asymptotic values were well matched by the $a_{1}^{u}, a_{2}^{u}$ and $a_{1}^{v}$ time coefficients. Figure 1 also shows the reconstructed components of velocity at point A illustrated in Fig. 2. Note that the reconstructed velocities $u$ and $v$ using the Poddec data did not agree well with the results of MFIX because herein only 4 and 3 modes were used for $u$ and $v$, respectively. The difference between the MFIX and the ODEx solution was attributed to the fact that the ODEx results were obtained from the momentum equations only and did not take into account the pressure correction equation.

Figure 3 shows the variation of the cumulative sum of energy of the POD modes corresponding to the $u$ and $v$ velocity for the case s3b. The velocity variation at points A and $\mathrm{B}$ is shown in Figs. 4 and 5 for different numbers of POD modes. It is apparent from these results that the accuracy of the low order model increases as the number of POD modes increases.

As previously mentioned, three computer codes were developed to reduce the governing PDEs to a smaller set of ODEs. The good agreement between the results of the three codes, ODEx, ODExNL and ODExMIN, indicated that it was quite likely that each of these three methods were correctly implemented. The assumption made was that the differences noticed between the solution of the system of PDEs and the reconstructed solution was due to the level of errors in the PDE solver. In order to verify this assumption, the analysis has been applied to a simpler case, a non-homogeneous Burgers equation.

The solution of the Burgers equation has been calculated for different levels of tolerances 
imposed in the solver. The tolerance was defined as

$$
\text { tol }:=\left|\frac{u^{n}-u^{n-1}}{u^{n}}\right|,
$$

where $u^{n-1}$ and $u^{n}$ denote results of previous and current iterations. For each tolerance level, the error values, $\epsilon$, were computed as

$$
\epsilon:=\left|\frac{L H S-R H S}{\sqrt{L H S^{2}+R H S^{2}}}\right| .
$$

\begin{tabular}{|c|c|}
\hline TOLERANCE & ERROR \\
\hline $0.01 \%$ & $0.78 \%$ \\
$0.3 \%$ & $4.8 \%$ \\
$5 \%$ & $17.3 \%$ \\
$50 \%$ & $30.5 \%$ \\
\hline
\end{tabular}

Table 3: Tolerance and error levels in Burgers equation.

The tolerance levels and the corresponding error levels are shown in Table 3. For the imposed tolerance of $0.01 \%$, a very good agreement has been obtained between the reconstructed solution (using ODEx) and the direct solution, as shown in Fig. 6. The differences between the reconstructed solution and the direct solution increased when the tolerance was $5 \%$ or higher.

The error analysis of the Burgers equation has been applied to a system of two coupled PDEs called the "double Burgers", $u_{t}+v u_{x}=f(x, t)$ and $v_{t}+u v_{x}=g(x, t)$. The conclusions resulted from the Burgers equations extended to the "double Burgers" equations. These results were presented at the Multiphase Fluid Dynamics Research Consortium meeting in Columbia, MD, September 2002.

Once the influence of the local accuracy of the PDEs on the reconstructed solution was proven on the Burgers equation, an attempt was made to improve the local error in MFIX. Consequently, the case3b was run using MFIX with a new convergence criteria: $(R H S-L H S) / \sqrt{R H S^{2}+L H S^{2}}$. The averaged error decreased from $17 \%$ to $1 \%$. The variation of time coefficients $a_{v}(t)$ improved significantly. The time coefficients of the $v$ velocity captured correctly the long term solution.

The low order model based on proper orthogonal decomposition applied to case s3b (momentum conservation equations of the fluid phase, without solid phase) reduced 6000 PDEs to 26 ODEs. The computational CPU time was reduced from 600 seconds to 30 seconds.

The results of ODEx applied to cases s3a and s3b did not agree well with the MFIX solutions. Thus, we decided to follow the algorithm of MFIX and developed a code called ODExMFIX. In ODExMFIX, the discretized momentum equations and pressure correction 
equation, which are systems of linear equations, were projected to the basis functions to obtain the reduced-order systems of linear equations. Then an iterative procedure which is similar to the algorithm of MFIX was used to solve these reduced-order systems of linear equations. First, the reduced-order systems for momentum equations were solved based on the current time coefficients of pressure. Then the reduced-order system for pressure correction was solved to obtain the correction for the time coefficients of pressure. Finally the time coefficients for velocities and pressure were corrected.

The results corresponding to case s3c using ODExMFIX are shown in Figs. 7 and 8. The number of POD modes used for the reconstruction of pressure, $u$ and $v$ was 15, 23 and 16, respectively. The POD modes captured $99.99 \%$ of the energy spectrum. The velocity variation at the points $\mathrm{A}$ and $\mathrm{B}$ shown in Figs. 7 and 8 proves that the accuracy of the low order model based on ODExMFIX increased compared to the low order models based on ODEx, ODExNL and ODExMIN. This task was completed by Dr. Paul Cizmas of the Aerospace Engineering Department at Texas A\&M University.

\subsection{Task 3}

The work on the visualization tools for Galërkin ODEs has been started. This included visualization of reconstructed flow motion, and visualization of flow motion in phase space through POD time coefficients. Using POD spatial modes, visualizations of the reconstructed flow motion were generated. POD amplitude coefficients were used to create animations of the temporal behavior of the flow in phase space. Part of these results were presented at the Multiphase Fluid Dynamics Research Consortium meeting in Columbia, MD, September 2002 and at the "Vision 21" meeting held at Iowa State University in November 2002. This task was completed by Dr. Antonio Palacios of the Mathematics Department at San Diego State University.

\section{Conclusions}

The project is progressing as planned, on time and budget. Several challenges were overcame on the development of the algorithm for the order reduction. We ended up developing more than one implementation for the low order model. The ODEx, ODExNL and ODExMIN codes produce an economical low order model. The ODExMFIX code produces a more computational intensive but more accurate low order model. The visualization tools were developed as planned. 

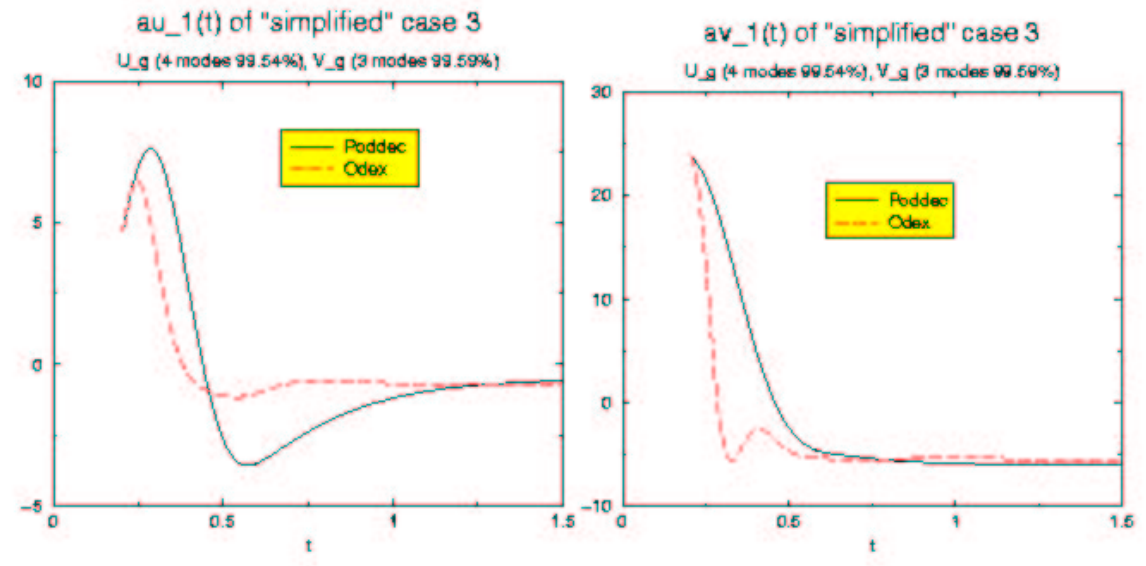

au_2(t) of "simplified" case 3

av_2(t) of "simplified" case 3

U 14 modes $99.54 \%), V \_g$ (3) modes $\left.98.59 \%\right)$

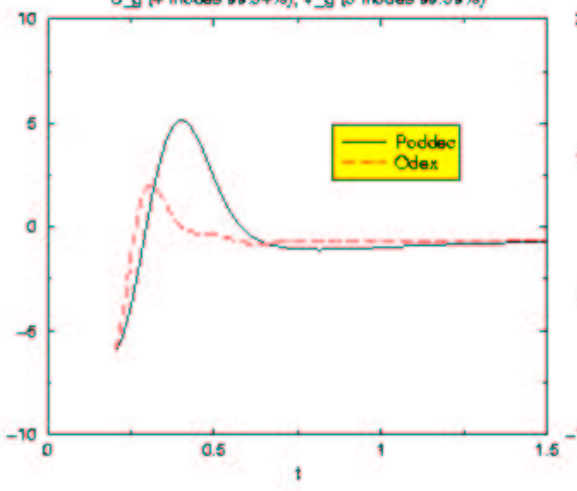

$\mathrm{U}$ S s 4 modes $98.549 \%$, V_st 3 modes $90.59 \%$ )

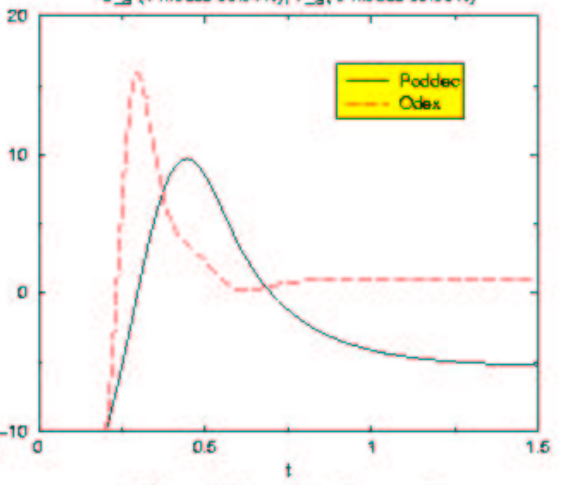

$V_{\text {g }}$ of "simplified" case 3

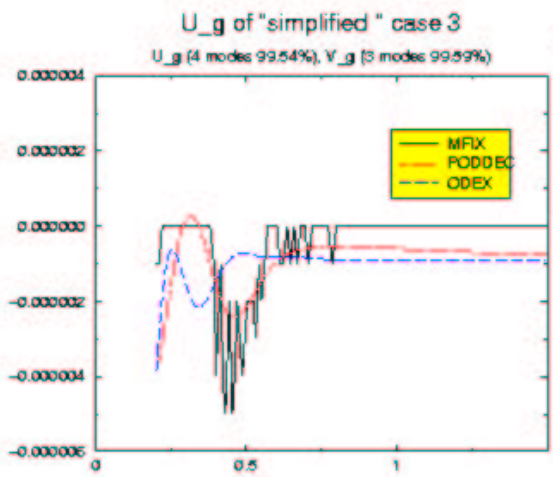

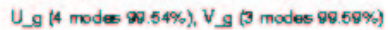

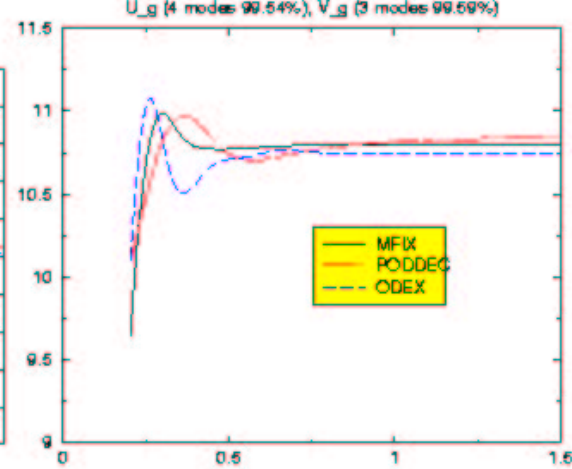

Figure 1: Time coefficients and reconstructed solutions with ODEx 

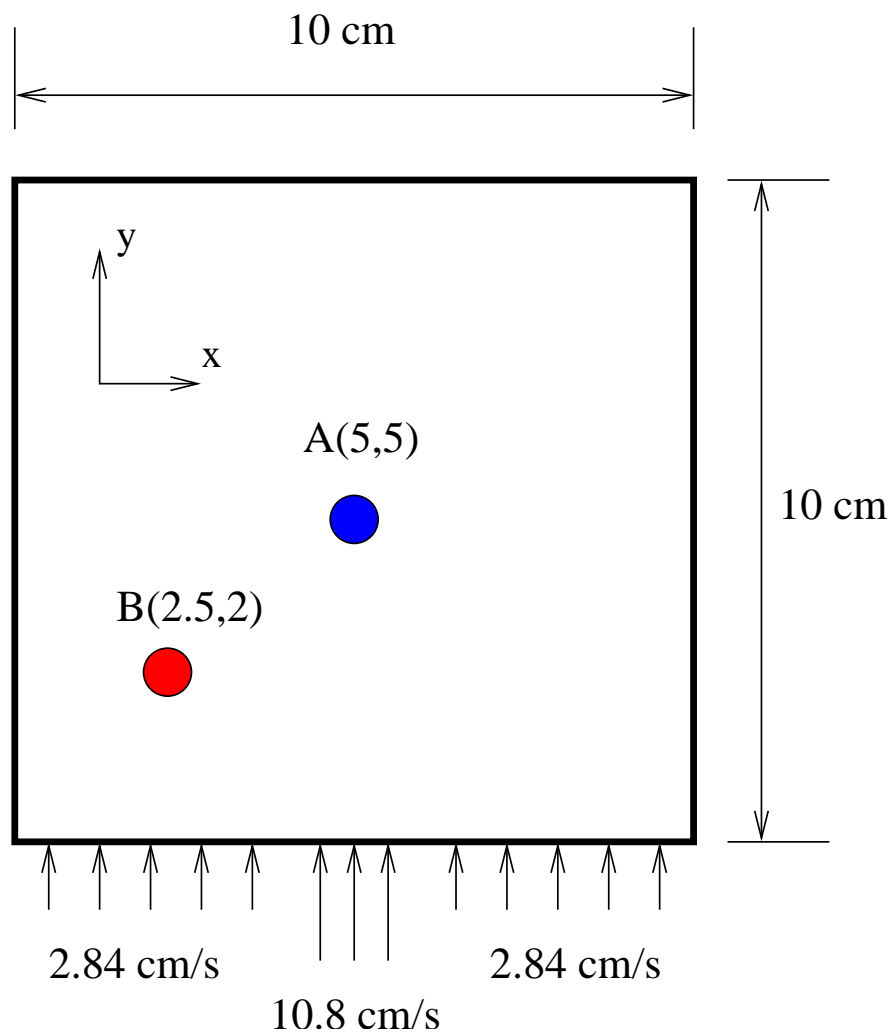

Figure 2: Geometry and boundary conditions for case s3b.
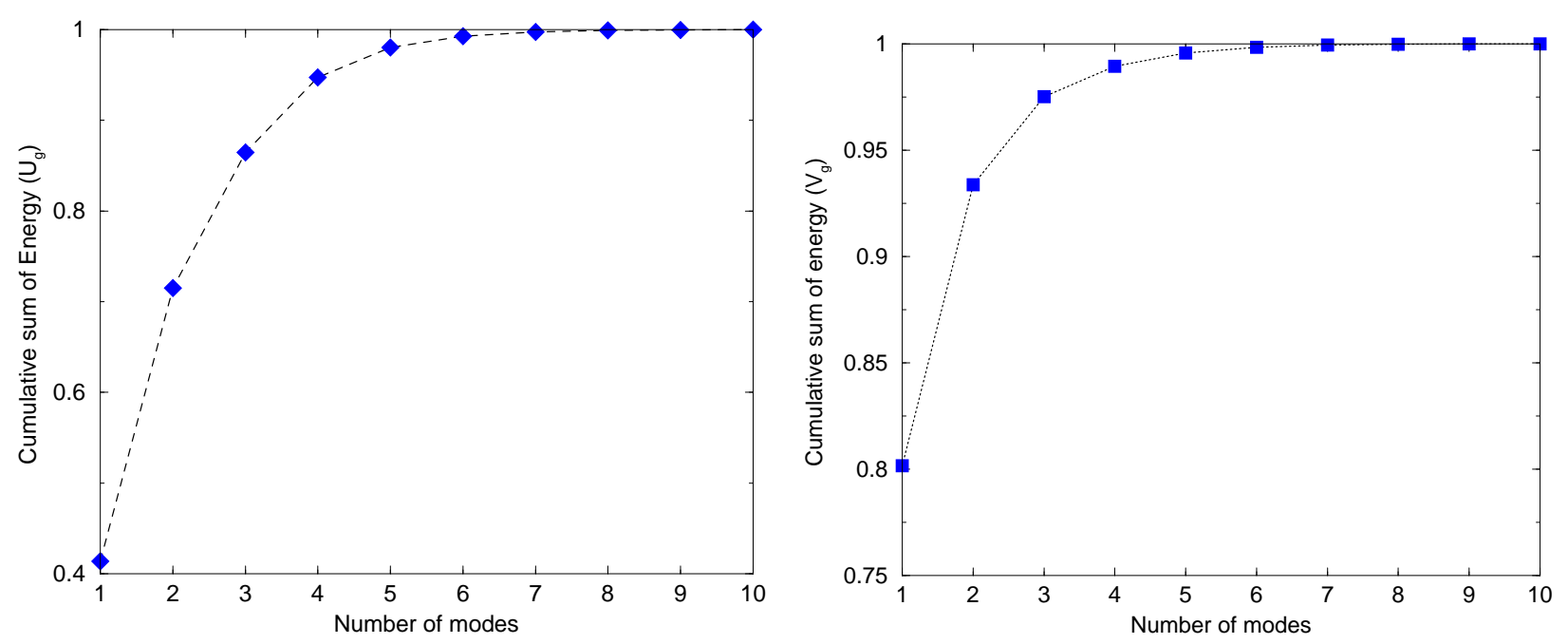

Figure 3: Cumulative sum of energy of POD modes for case s3b. 

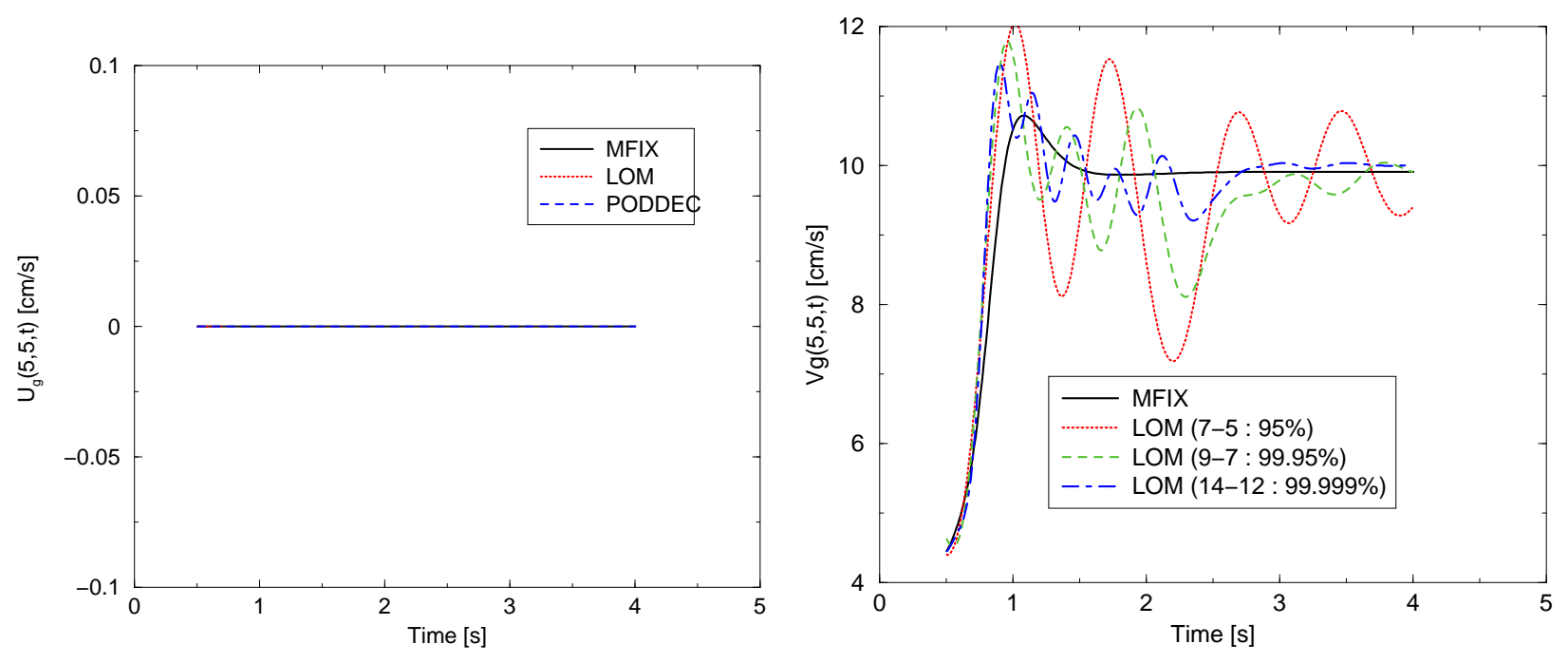

Figure 4: Velocity variation at point A, case s3b.
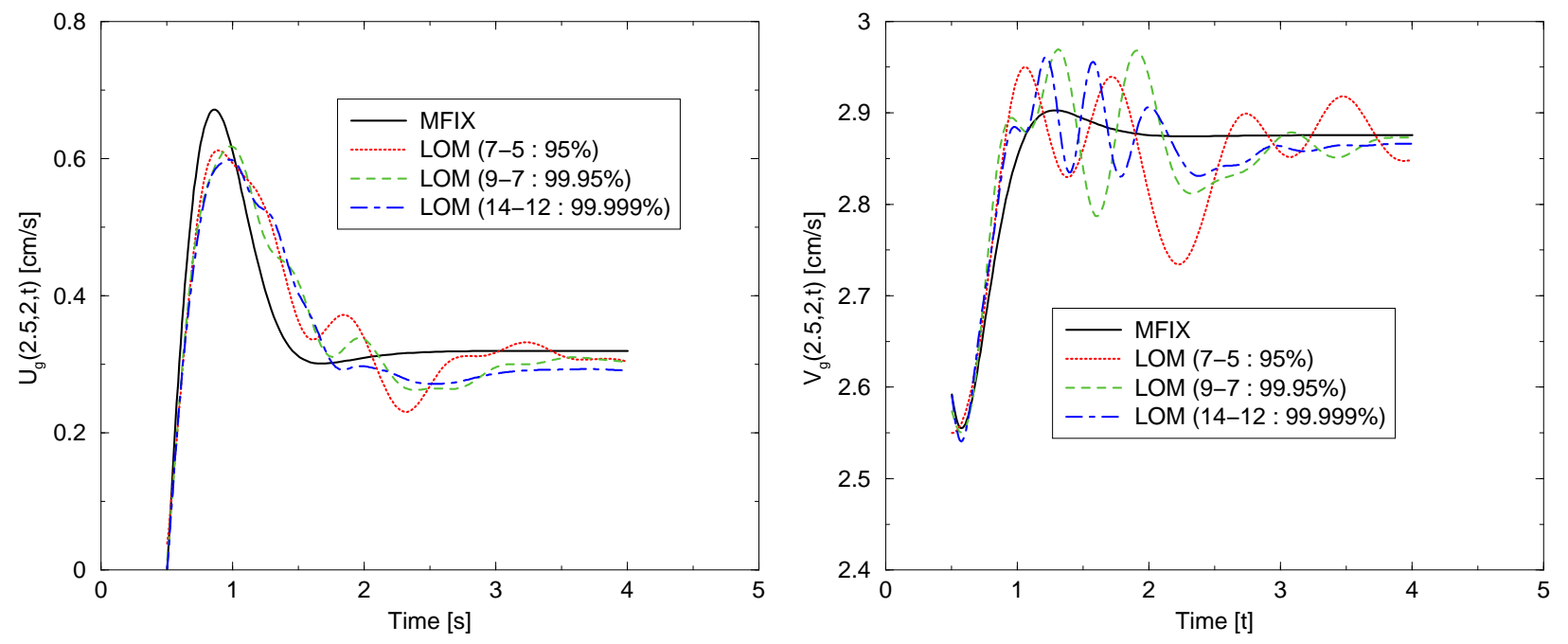

Figure 5: Velocity variation at point B, case s3b. 


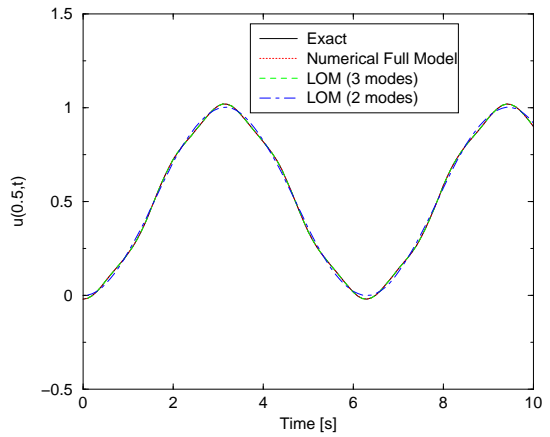

(a) Tolerance $0.01 \%$

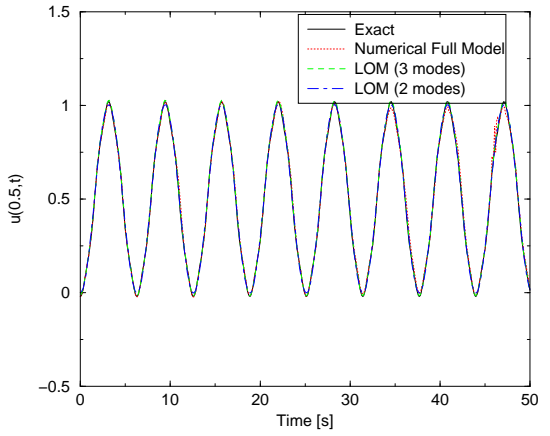

(b) Tolerance $5 \%$

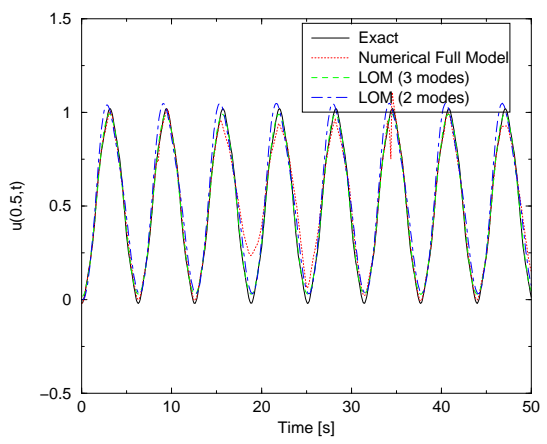

(c) Tolerance $50 \%$

Figure 6: Reconstructed $u(0.5, t)$ at different tolerance levels. 

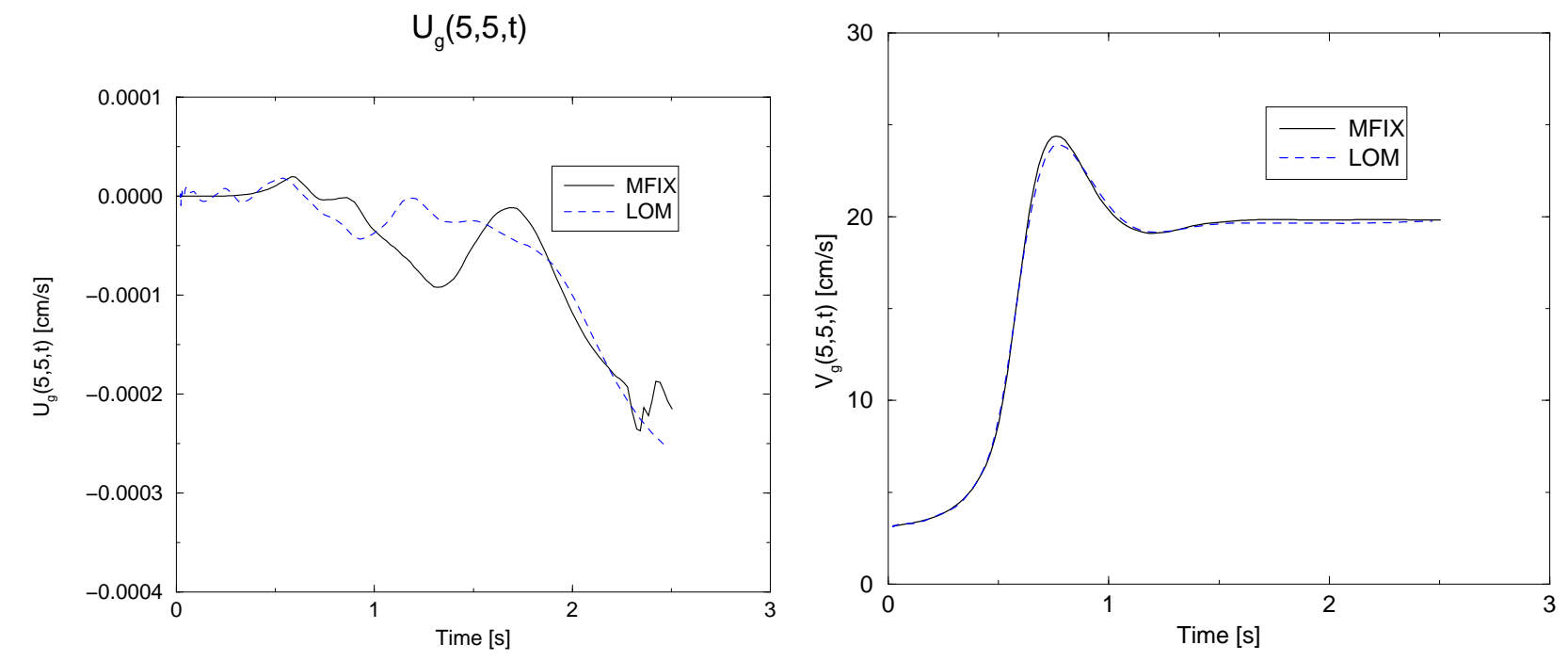

Figure 7: Velocity variation at point A, case s3c.
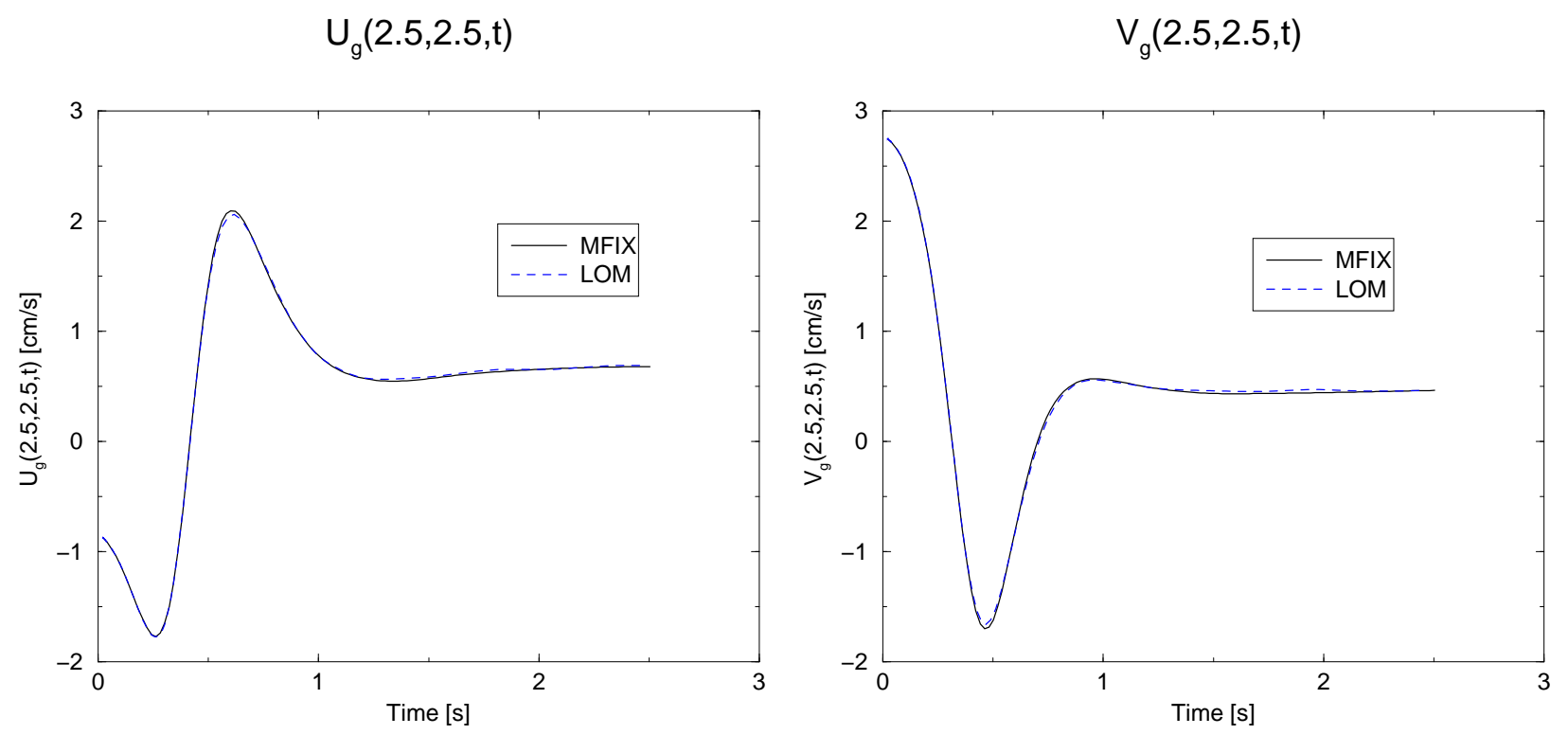

Figure 8: Velocity variation at point $B$, case s3c. 